\title{
Extracción, Caracterización Parcial y Termoestabilidad de la Enzima Transglutaminasa en Surimi, en Músculo Blanco de Jurel y en Miofibrillas de Carne de Vacuno
}

Emilia M. Curotto(1) ${ }^{(1)}$ Marta L. Dondero ${ }^{(2)}$, Cristián J. Muñoz ${ }^{(1)}$ y Lorena A. Álvarez ${ }^{(1)}$

(1) Instituto de Química, Laboratorio de Enzimología, (2) Escuela de Ingeniería en Alimentos, Pontificia Universidad Católica de Valparaíso, Avda. Brasil 2950, Valparaíso-Chile

\begin{abstract}
Resumen
El objetivo de esta investigación fue determinar la presencia de transglutaminasa en los extractos de surimi, músculo blanco de jurel (Trachurus murphyi) y en miofibrillas de carne de vacuno. Esto, con el fin de evaluar su termoestabilidad y los parámetros cinéticos más relevantes para proyectar la utilización de estos tejidos en la industria de nuevos productos alimentarios. Las actividades de transglutaminasa fueron $4.7 \times 10^{-3}, 13.0 \times 10^{-3}$ y $53.0 \times 10^{-3} \mathrm{Ul} / \mathrm{g}$ tejido para surimi, músculo blanco de jurel y carne de vacuno, respectivamente. Se caracterizó parcialmente la transglutaminasa en miofibrillas, encontrándose valores de pH óptimo de 6.5 y $40^{\circ} \mathrm{C}$ de temperatura. Se postula la existencia de dos transglutaminasas en el músculo blanco de jurel, con valores óptimos de pH de 5.4 y 7.6, temperaturas de 50 y $60^{\circ} \mathrm{C}$ y concentraciones de calcio de 10 y $40 \mathrm{mM}$. Para surimi los valores encontrados son semejantes a los de músculo blanco de jurel. Se realizaron pruebas de termoestabiliad, obteniéndose valores de las constantes de inestabilidad entre $3.3 \times 10^{-3}$ y $0.4 \mathrm{~h}^{-1}$ a las distintas temperaturas de preincubación. Los valores de las energías de activación de la inactivación se encuentra entre 12.46 y $39.37 \mathrm{KJ} \mathrm{mol}^{-1} \mathrm{oK}^{-1}$.
\end{abstract}

\section{Extraction, Partial Characterization and Thermostability of Transglutaminase of Surimi and Jack Mackerel White Muscle and for Myofibrils of Bovine Meat}

\begin{abstract}
The objective of this research was to carry out the extraction and characterization of surimi and jack mackerel white muscle (Trachurus murphyi) and of bovine meat and to determine the thermostability of transglutaminase. Kinetics parameters and thermostability are more relevant to project the utility of this tissue in new food products development. The activities of this enzyme were of $4.7 \times 10^{-3}, 13.0 \times$ $10^{-3}$ y $53.0 \times 10^{-3} \mathrm{Ul} / \mathrm{g}$ tissue for surimi and white muscle of jack mackerel, and meat of bovine respectively. It characterized transglutaminase of bovine meat, finding values of optimal $\mathrm{pH}$ of 6.5 and $40^{\circ} \mathrm{C}$ of temperature. The existence of two transglutaminases was postulated in the white muscle of jack mackerel, with optimal $\mathrm{pH}$ values of 5.4 and 7.6, temperatures of 50 and $60^{\circ} \mathrm{C}$, and calcium concentrations of 10 and $40 \mathrm{mM}$. Inactivation constants ranged between $3.3 \times 10^{-3}$ and $0.4 \mathrm{~h}^{-1}$ for different temperatures of preincubation. The activation energy of denaturation was about $12.46 \mathrm{y}$ 39.37 KJ mol${ }^{-1} \mathrm{oK}^{-1}$.
\end{abstract}

Keywords: transglutaminase, thermostability, inactivation constant, inactivation energy 


\section{INTRODUCCION}

La enzima Transglutaminasa cataliza la reacción de acil transferencia entre el grupo $\gamma$-carboxiamida y residuos de glutamina presentes en proteínas, polipéptidos y numerosas aminas primarias. Cuando el grupo $\varepsilon$-amino de lisina actúa con el residuo glutamil como un ácil aceptor, se forma un enlace entrecruzado $\varepsilon$-( $\gamma$-glutamil)-lisina inter o intra-molecular entre las cadenas de proteínas (J.E. Folk et al,1973, 1977, 1980). Las transglutaminasas están ampliamente distribuídas en numerosos organismos, tejidos, fluídos corporales, incluyendo vertebrados, invertebrados, plantas y microrganismos (I. Kumazawa et al., 1997; H. Kishi et al., 1991; I. Icekson et al., 1987; H. Ando et al., 1989). Posee una característica importante que la distingue de las otras enzimas utilizadas en la industria alimentaria; permite la polimerización de sustratos proteicos en productos de mayor peso molecular (Revista Prinal 2000). Existen estudios de esta enzima en mamíferos, peces y muy pocos en plantas (P Nielsen, et al., 1995).

Esta enzima se ha utilizado para catalizar los entrecruzamientos entre ciertas proteínas, como proteínas de suero, soja, gluten, miosina y actomiosina entre otras. La modificación de las proteínas de los alimentos por transglutaminasa mejora la textura de los productos, ayuda a proteger a la lisina de reacciones químicas adversas, encapsula los lípidos, forma films resistentes al calor y agua, mejora la elasticidad y capacidad de retención de agua, modifica la solubilidad y propiedades funcionales de las proteínas, se forman proteínas con un alto valor nutritivo a través del entrecruzamiento de los enlaces entre ellas. (Y. Zhu et al., 1995, M. Dondero et al., 2002, 2006).

Dado las consideraciones anteriores, se ha estudiado la presencia de transgluminasa endógena en varias especies marinas (Sardina pilchardus) (Batista et al., 2000) ostra japonesa (Cassostrea gigas) (Kumazawa et al., 1997, hígado de Walleye Pollack (Theragra chalcogramma), (Kumazawa et al., 1996), entre otras, las cuales son extraídas, seguidas de purificación parcial y caracterización cinética. Se ha reportado también que la transglutaminasa endógena presente en el surimi de Alaska pollack (Theragra chalcogramna) congelado reacciona durante la confección del kamabobo y contribuye a la polimerización de las cadenas pesadas de la miosina, aumentando el número de enlaces $\varepsilon$-( $\gamma$-glutamil)-lisina, favoreciendo la fuerza de gel, la que disminuye si se agregan inhibidores de esta enzima (N. Seki et al., 1990; J. Wan et al., 1994; Y. Kumazawa et al., 1995).

Debido a éstos antecedentes, es posible comparar esta información con transglutaminasas endógenas provenientes de otros orígenes aumentando así, el conocimiento en otras especies. El objetivo de esta investigación es obtener la enzima transglutaminasa presente en: a) surimi, b) el músculo blanco del jurel (Trachurus murphyi), c) miofibrillas de carne de vacuno; caracterizar sus propiedades cinéticas y evaluar la termoestabilidad para proyectar su aplicación en la generación de nuevos productos de carne de vacuno entrecruzado con surimi o con músculo blanco de jurel (Trachurus murphyi).

\section{MATERIALES Y MÉTODOS.}

\section{Materia Prima}

El Jurel (Trachurus murphyi), fue obtenido fresco en las Caletas de pescadores de "Portales" y el "Membrillo" de la ciudad de Valparaíso. El surimi de jurel fue proporcionado por la empresa pesquera "El Golfo" de Talcahuano, VIII Región, Chile. Como crioprotectores fueron adicionados sacarosa-sor bitol al $8 \% 1: 1$ y $0,25 \%$ de tripolifosfato de sodio y tetrapirofosfato de sodio $1: 1$. La carne absolutamente desgrasada fue suministrada por la Escuela de Ingeniería en Alimentos de la Pontificía Universidad Católica de Valparaíso, Chile. La transglutaminasa microbiana (TG-BH) fue proporcionada por Prinal S.A.

\section{Obtención de los Extractos Crudo.}

Se realizó el homogeneizado de la muestra (surimi de jurel, músculo blanco de jurel, miofibrillas de vacuno) con bicarbonato de sodio $60 \mathrm{mM},\left(\mathrm{pH}\right.$ 9.6), en una relación 1:1, a $4{ }^{\circ} \mathrm{C}$. Luego, se prensó a través de gasa y el filtrado se pasó por lana de vidrio. Posteriormente, este filtrado se centrifugó a 
$11.600 \times \mathrm{g}$ a $0^{\circ} \mathrm{C}$ durante 20 minutos. Al sobrenadante se le midió actividad transglutaminasa y concentración de proteínas.

\section{Concentración del Extracto Crudo.}

El extracto crudo se concentró mediante una precipitación fraccionada con sulfato de amonio al 30\% y $80 \%$ de saturación. Se dejó durante $24 \mathrm{~h}$ a $0^{\circ} \mathrm{C}$. El precipitado se resuspendió en el volumen mínimo de tampón Tris-Acetato $20 \mathrm{mM}$, pH 6.0. El precipitado redisuelto se centrífugó a 11.600 × g durante 15 min, obteniéndose un extracto clarificado.

\section{Purificación parcial de Transglutaminasa}

Se realizaron purificaciones utilizando dos columnas cromatográficas, una de intercambio iónico DEAE-Sepharose CL-6B y otra de exclusión molecular, Sephacryl S-100-HR. Para ambas cromatografías se utilizó como base de elución tampón acetato $\mathrm{pH}=5,0 ; 0,1 \mathrm{M}$. La cromatografía aniónica se realizó con un gradiente salino de 0 a $1 \mathrm{M}$ de $\mathrm{NaCl}$.

\section{Medida de la Actividad Enzimática.}

Se coloca en un tubo de ensayo 200 uL de tampón Tris-Acetato 0.1M, pH 6.0. 150 uL de CBZ-Lglutamilglicina, $50 \mathrm{uL}$ de hidroxilamina y se incuba a $37^{\circ} \mathrm{C}$, durante $5 \mathrm{~min}$. Luego se agregan $500 \mathrm{uL}$ de la muestra que contiene la enzima y se incuba durante $30 \mathrm{~min}$. A continuación, se añade $1 \mathrm{~mL}$ de ácido tricloracetico al 15\% con cloruro ferrico. Se enfria y centrifuga a $7.500 \times$ g durante $10 \mathrm{~min}$.

En el sobrenadante, se mide la actividad enzimática. El valor de la concentración de producto, se obtiene interpolando en una curva de calibrado de Lácido glutámico $\gamma$ - monoácido hidroxámico entre 0.1- $0.8 \mathrm{mM}$ (Ajinomoto 1998). Las lecturas se realizan en un espectrofotómetro a $525 \mathrm{~nm}$.

\section{Determinación de la Concentración de Proteínas}

Fue determinada utilizando el método de Lowry (1957). Se usó como estandar, la proteína albumina de suero de bovino (BSA).

\section{Determinación de Temperatura de ensayo, pH óptimo y Efecto de la Concentración de Ca ${ }^{+2}$.}

La temperatura de ensayo fue determinada entre $20-70^{\circ} \mathrm{C}$, para todas las transglutaminasas de diferente origen. El pH óptimo se determinó entre 4.5-8.7 a fuerza iónica constante, utilizando tampón Good 0,1 M. El efecto del ion $\mathrm{Ca}^{+2}$ en la actividad enzimática, se determinó utilizando una solución de cloruro de $\mathrm{Ca}^{+2}$ entre $0-100 \mathrm{mM}$. a pH óptimo y temperatura determinada en los ensayos previos. Los ensayos de actividad enzimática fueron realizados de acuerdo a la metodología descrita anteriormente.

\section{Electroforesis en Gel de Poliacrilamida-SDS.}

SDS-PAGE fue realiza siguiendo la metodología de Laemmli (1974). Corriente constante a 20mA en el gel concentrador (3\%), aumentando a $30 \mathrm{~mA}$ en el gel separador (10\%). Los marcadores de peso molecular utilizados están entre 205.000-29.000 dalton. ( Sigma Chemical Co).

\section{Estabilidad Térmica}

La actividad remanente de las diferentes transglutaminasas, fue determinada a distintos intervalos de tiempo después de preincubar a diferentes temperaturas. $\left(30-50^{\circ} \mathrm{C}\right)$, utilizando tampón Tris-Acetato $20 \mathrm{mM}$, pH 5.5. El ensayo de actividad enzimático fue realizado en las condiciones descritas anteriormente. La constante de inestabilidad $\mathrm{ki}$, fue determinada aplicando la ecuación monoexponencial de primer orden, $A=A_{0} * e^{-k t}$ (Greco y col. 1993). La energía de inactivación fue calculada según la ecuación de Arrehnius. 


\section{RESULTADOS Y DISCUSIÓN.}

Los ensayos fueron realizados en los extractos crudos concentrados, de surimi, músculo blanco de jurel y miofibrillas de carne de vacuno. Los resultados se muestran en la Tabla 1. La actividad de transglutaminasa en surimi, fue bastante más baja que en las otras especies. Esto puede ser atribuido a que el surimi es un producto ya manufacturado a diferencia de las otras muestras.

\section{Caracterización de la Transglutaminasa del músculo blanco de Jurel}

Se estudiaron algunas propiedades cinéticas de la acción de la transglutaminasa de músculo de jurel, de surimi y de miofibrillas de vacuno importantes, para la formación de geles en mezclas de surimi y miofibrillas de vacuno y músculo blanco de jurel. Los ensayos realizados permitieron la obtención del $\mathrm{pH}$, temperatura y concentración de calcio óptima para las transglutaminasas analizadas.

Tabla 1. Actividades de las transglutaminasas en los extractos de surimi de jurel, músculo blanco de jurel, y miofibrillas de vacuno.

\begin{tabular}{|l|l|l|l|l|}
\hline $\begin{array}{l}\text { Extracto } \\
\text { crudo }\end{array}$ & Actividad $\times 10^{3} \mathrm{Ul} / \mathrm{mL}$ & Proteina $\mathrm{mg} / \mathrm{mL}$ & $\begin{array}{l}\text { Actividad Específica } \times \\
10^{5} \mathrm{Ul} / \mathrm{mg} \text { proteína }\end{array}$ & $\begin{array}{l}\text { Actividad Específica } \times 10^{3} \\
\mathrm{Ul} / \mathrm{g} \text { tejido }\end{array}$ \\
\hline Surimi & 10,62 & 336,19 & 3,16 & 4,66 \\
\hline Jurel & 21,65 & 245,61 & 8,82 & 13,00 \\
\hline Miofibrillas & 50,97 & 141,98 & 35,90 & 52,95 \\
\hline
\end{tabular}

Los resultados para el músculo blanco de jurel se presentan en las figuras 1 a 3 . El análisis de Gauss para la curva de actividad y $\mathrm{pH}$, permite postular la existencia de dos transglutaminasas en el músculo blanco de jurel cuyos valores de $\mathrm{pH}$ son $5,66 \pm 1,58$ y 7,66 $\pm 0,8$ con un valor de $\chi^{2}$ de aproxi madamente cero. Y. Kumasawa (1997), por su parte encontró una actividad relativa a pH 8,0 para las transglutaminasas presentes en Ostra Japonesa. Comparativamente, el valor encontrado en esta investigación, se encuentra dentro de lo sugerido por G. Tsai et al., (1996), para la transglutaminasa de Streptoverticillium lada- kanum que presenta la más alta actividad entre pH 6,0 y 7,0 .

El análisis de Gauss para la curva de actividad y temperatura también muestra dos valores, 44,76 \pm $7,94{ }^{\circ} \mathrm{C}$ y $56,84 \pm 11,44{ }^{\circ} \mathrm{C}$., con un valor de $\chi^{2}$ aproximadamente de cero (Figura 2). Estos valores se pueden contrastar con lo encontrado por Y. Kumasawa et al., (1997) quién determinó la temperatura óptima para las transglutaminasas endógenas de Ostra Japonesa a pH 8,5 de 40 y $25^{\circ}$ C. Además, A. Yasueda et al.,(1994) informan que en hígado de besugo del mar rojo, la máxima actividad fue a los $55^{\circ} \mathrm{C}$. La actividad máxima para transglutaminasas encontrada por A. Nowsad (1996), en Alaska Pollack , Horse mackerel, Pacific mackerel y Spanish mackerel fue entre los 20-25 ${ }^{\circ} \mathrm{C}$.

El efecto del ión $\mathrm{Ca}^{+2}$ según los gráficos de Gauss, fueron de 8,0 \pm 0,81 y 42,63 \pm 15,67 mM $\left(\chi^{2}\right.$ igual a cero) para las dos enzimas de músculo blanco de jurel (Figura 3). Y. Kumasawa (1997), por su parte encontró la máxima actividad a concentraciones mayores a $100 \mathrm{mM} \mathrm{Ca}^{+2}$, para la enzima transglutaminasa 1 y valores de $25 \mathrm{mM}$ para su isoenzima 2, en Ostra Japonesa. En estudios de hígado de besugo del mar rojo, hepatocito de limulus e higado de cuy, la enzima purificada requiere concentraciones de 0,5; 8 y 10 mM respectivamente (Y. Kumasawa et al., 1997), (H. Lee et al.1997), demostró además que la cantidad máxima de adición de calcio a transglutaminasa de surimi fue de $250 \mathrm{mM}$, de lactato de calcio ó $0.125 \mathrm{mM}$, de acetato de calcio. Los resultados obtenidos con surimi de jurel, fueron semejantes a los datos que,se obtuvieron con el músculo blanco de jurel, lo que es razonable ya que el surimi utilizado proviene del músculo blanco del jurel.

\section{Purificación Parcial de Transglutaminasa de Miofibrillas de Carne de Vacuno}

Se realizaron dos experiencias cromatográficas de purificación a partir del extracto crudo concentrado, obteniendo un factor de purificación de 27.4 veces y una actividad específica de $99 x$ 
$10^{-4} \mathrm{UI} / \mathrm{mg}$ proteina, $1 \times 10^{-4} \mathrm{UI} / \mathrm{g}$ miofibrillas. Además, los resultados fueron evaluados por Electrofóresis PAGE-SDS (Figura 4). Esto se realizó para comparar en este tipo de tejido, las variaciones que se podrían encontrar entre la transglutaminasa parcialmente purificada con la información obtenida del extracto crudo.

Se realizó la electroforesis SDS-PAGE en gel separador al 12\%. Como se puede ver, en la electroforesis, existe una banda cercana a la de BSA la que se repite en los carriles 5, 6 y 7 , correspondientes a la transglutaminasa parcialmente purificada, lo que hace suponer que esta banda correspondería a la enzima analizada. Además, cuando se coloca mayor concentración de muestra en el carril 7, aparece una segunda banda que podría corresponder a otra isoforma, ya que en todos los casos analizados se detectan por lo menos dos de ellas.

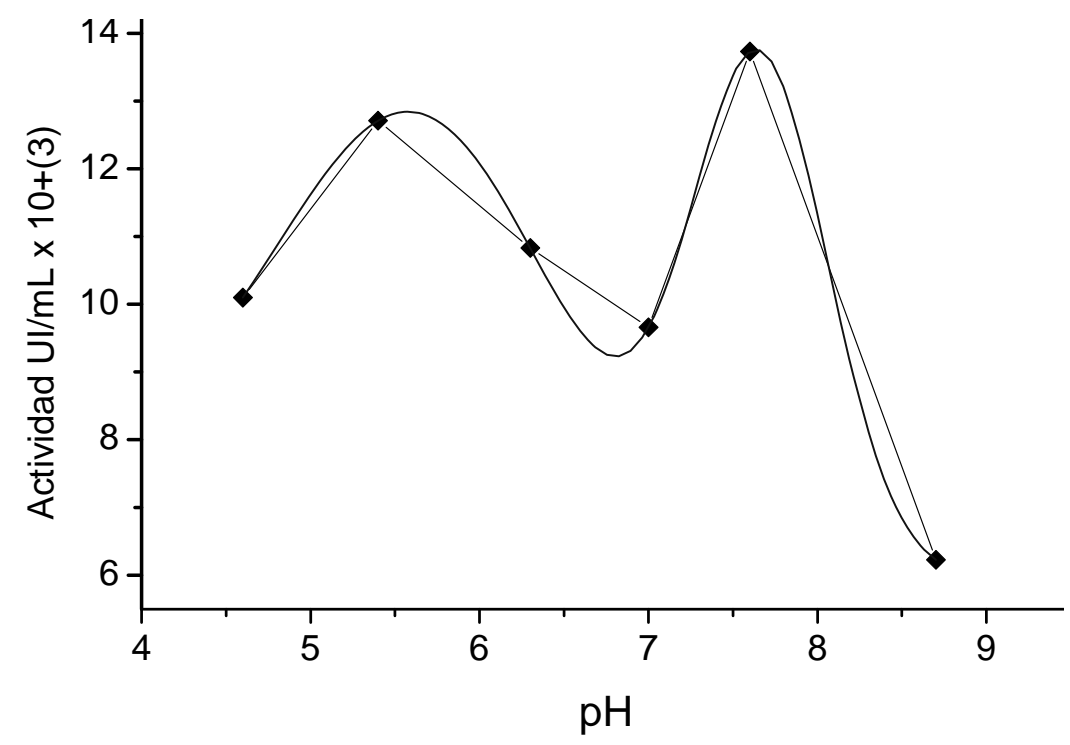

Fig 1. Actividad de transglutaminasa de músculo blanco de jurel en extracto crudo en función del pH, La actividad se determinó, utilizando diferentes pHs a partir de tampones Good $0.1 \mathrm{M}$ y $37^{\circ} \mathrm{C}$-—— (Experimental), —— Análisis de Gauss.

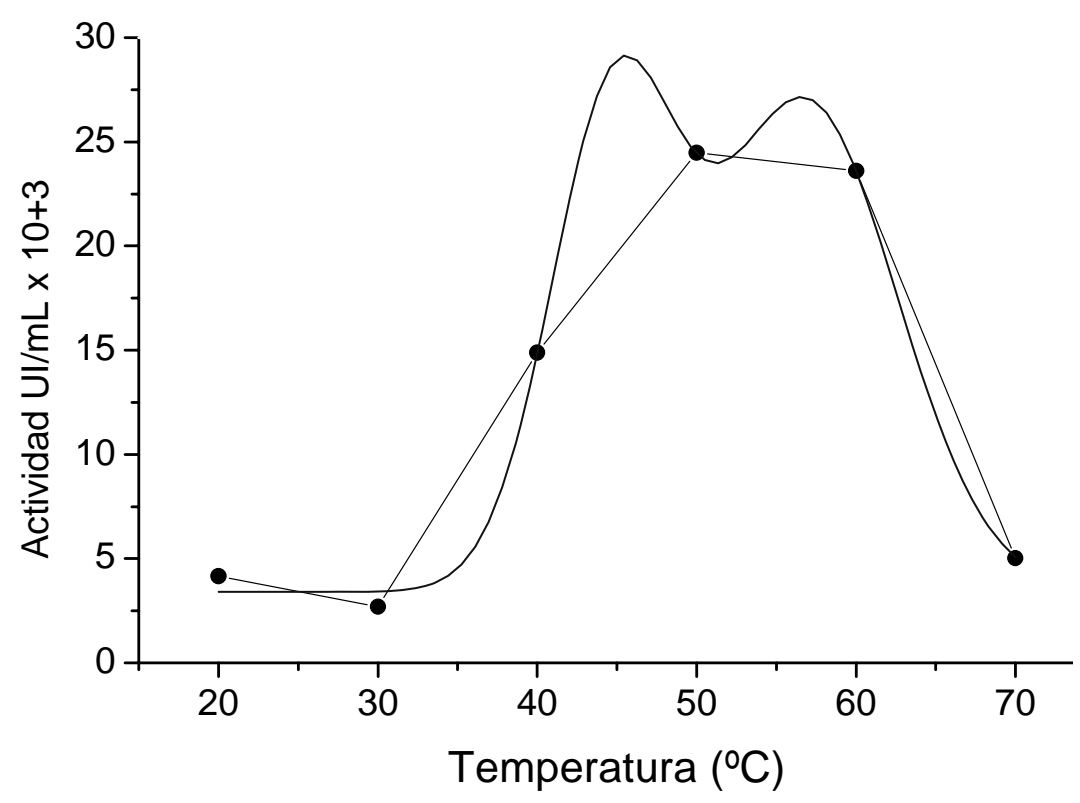

Fig. 2. Actividad de transglutaminasa de músculo blanco de jurel en extracto crudo en función de la temperatura, La actividad se determinó de acuerdo a la metodología de Ajinomoto, pH 6.0 La temperatura de ensayo se obtuvo mediante análisis de Gauss. —— (Experimental), — Análisis de Gauss. 


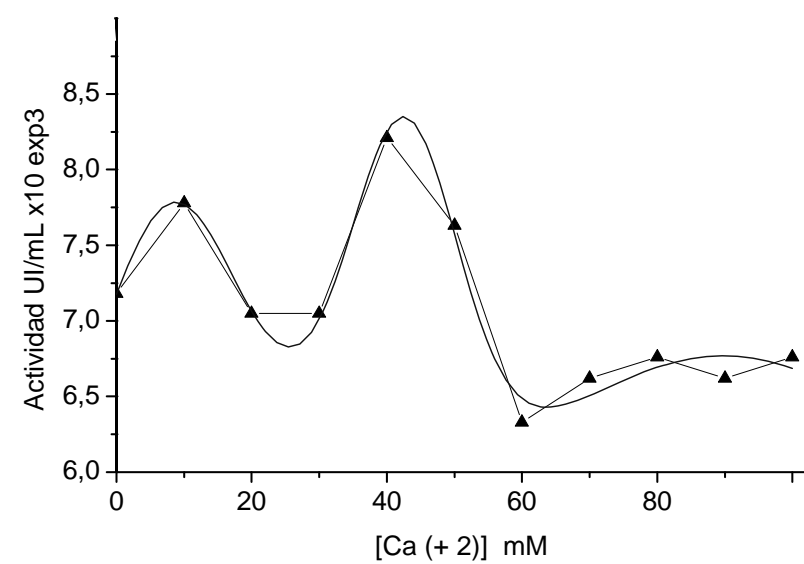

Fig. 3. Influencia de la concentración de calcio en la actividad de la transglutaminasa de músculo blanco de jurel. La actividad se determinó de acuerdo a la metodología de Ajinomoto a $37^{\circ} \mathrm{C}$. y pH 6.0. La determinación de las concentraciones de $\mathrm{Ca}^{+2}$ se realizó mediante análisis Gauss. _-_ (Experimental), - Análisis de Gauss.

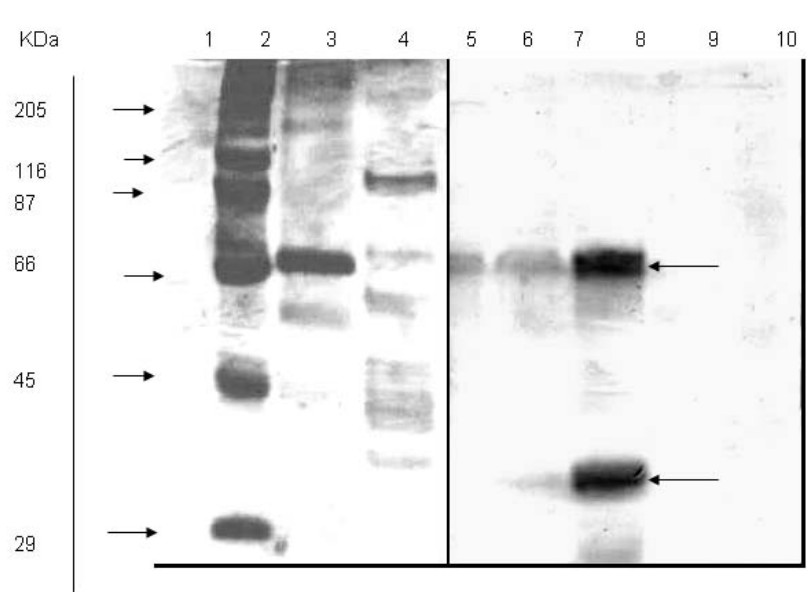

Fig. 4. Electroforesis PAGE-SDS. Carril 2 Estandares de peso molecular, carril 3 Transglutaminasa Sigma, carril 4 extracto crudo de miofibrillas, carril 5 y 6 extracto cromatográfico diluído, carril 7 extracto cromátografico concentrado. Carriles 8 y 9 , no se detecta muestras después de otras cromatografías.

\section{Caracterización de Transglutaminasa de miofibrillas de carne de vacuno.}

Esta caracterización se realizó con la transglutaminasa presente en el extracto crudo y con la enzima parcialmente purificada. La metodología empleada es semejante a los experimentos realizados con jurel y surimi. Los resultados se muestran en las figuras 5 y 6 . El análisis de Gauss para la curva de actividad versus pH dió un valor de $6,79 \pm 3,20$ y con un valor de $\chi^{2}$ de 9,01 para el extracto crudo y de $6,54 \pm 3,14$ con valor de $\chi^{2}$ de 0,12 . Se puede apreciar que no hay diferencias significativas en los valores de $\mathrm{pH}$ obtenidos entre la transglutaminasa del extracto crudo y la semipurificada. En esta última, el error es bastante menor. El gráfico de actividad de transglutaminasa versus temperatura en el extracto crudo de miofibrillas de vacuno, indica un valor de $39,8^{\circ} \mathrm{C}$ para el primer pico y de $60^{\circ} \mathrm{C}$ para el segundo. No presenta desviación en el análisis de Gauss y un $\chi^{2}$ igual a 0 . Para la transglutaminasa semipurificada se obtiene un valor de 41,8 $\pm 1,8$ ${ }^{\circ} \mathrm{C}$ por análisis de Gauss.

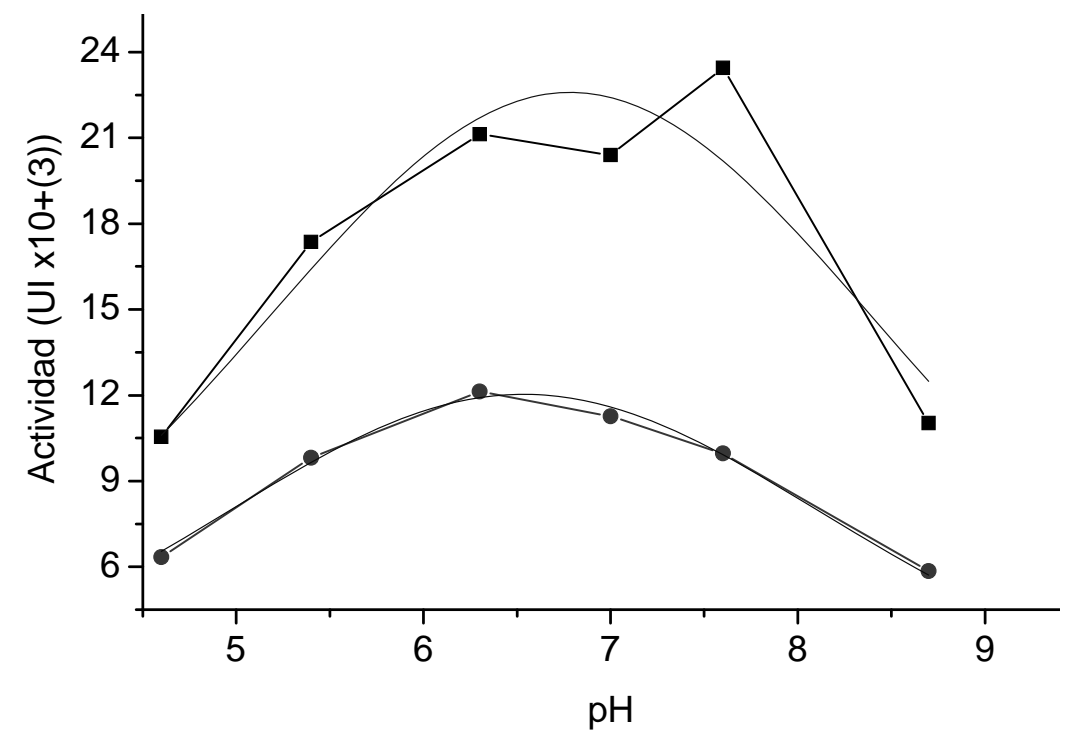

Fig.5. Actividad de transglutaminasa en función de $\mathrm{pH}$ en extracto crudo y purificado de miofibrillas de vacuno Se usó tampon Good, a fuerza iónica constante $(100 \mathrm{mM})$. La actividads enzimática se midió de acuerdo a Ajinomoto. Se aplicó análisis Gauss en la obtención de resultados.- - - (extracto crudo) $\longrightarrow$ - (extracto semipurificado). 
Calculo de la $k_{i}^{\prime}$ y de $E_{a}$ de la desnaturalización de los extractos respectivos

Los resultados se analizaron de acuerdo al modelo de decaimiento monoexponencial de primer grado, considerando que los puntos experimentales corresponden a velocidades iniciales, realizadas en los extractos crudos, para evitar: el efecto de contaminantes que pueden influir en la exactitud de las medidas. (A. Illanes 1994).

Del análisis de los gráficos se observa que en todos los casos, el comportamiento a $30^{\circ} \mathrm{C}$, las transglutaminasas muestran una constante de inestabilidad pequeña pero diferente para cada uno de los extractos analizados, indicando probablemente diferencias estructurales. Uno de los aspectos que cabe destacar, es el valor del tiempo de vida media de la enzima microbiana $(1,6 \mathrm{~h})$, a $50^{\circ} \mathrm{C}$ en cambio, todas las otras enzimas a igual temperatura, su tiempo de vida media fue mayor indicando una mayor estabilidad, favoreciendo la aplicación industrial de estas especies.

Para surimi de jurel, se observa que las curvas de 40 y $30^{\circ} \mathrm{C}$ casi se solapan indicando igual estabilidad a estas temperaturas; es probable que esto ocurra por efecto de los crioprotectores presentes en el surimi. Los valores de las constantes de inactivación de las transglutaminasas del músculo blanco del jurel fueron menores que las obtenidas en miofibrillas, aunque están dentro de los valores que se encuentran en bibliografía ( Fig.7 y Tabla 2).

S. Nury et al. ( 1990), calculó los parámetros de inactivación de la transglutaminasa de hígado de cerdo de guinea, mediante un modelo en donde la enzima se desnaturaliza teniendo un paso intermedio anterior de tipo $\mathrm{N} \rightarrow \mathrm{X} \rightarrow \mathrm{D}$, calculando dos constantes $\mathrm{k}_{\mathrm{i}}$, obteniendo un valor de energía de desnaturalización $\left(E_{a}\right)$ de 36,6 $\pm 3,4 \mathrm{~kJ} \mathrm{~mol}^{-1}$, similar al de 39,37 obtenido en esta investigación para miofibrillas de carne de vacuno (Tabla 3). También es importante destacar que la transglutaminasa microbiana no se ve afectada por la presencia de los crioprotectores, sacarosa-sorbitol, obteniéndose valores de $k_{i}^{\prime}$ semejantes.

Tabla 2. Valores de constante de inestabilidad aparente y tiempo de vida media de las transglutaminasas

\begin{tabular}{|c|c|c|}
\hline \multicolumn{3}{|c|}{ Microbiana } \\
\hline Temperatura $\left({ }^{\circ} \mathrm{C}\right)$ & $\mathrm{k}_{\mathrm{i}}\left(\mathrm{h}^{-1}\right)$ & $t_{1 / 2}(h)$ \\
\hline $50^{\circ} \mathrm{C}$ & 0,413 & 1,677 \\
\hline $40^{\circ} \mathrm{C}$ & 0,080 & 8,661 \\
\hline $30^{\circ} \mathrm{C}$ & 0,051 & 13,684 \\
\hline \multicolumn{3}{|c|}{ Miofibrillas de vacuno } \\
\hline Temperatura $\left({ }^{\circ} \mathrm{C}\right)$ & ki $\left(h^{-1}\right)$ & $t_{1 / 2}(h)$ \\
\hline $50^{\circ} \mathrm{C}$ & 0,150 & 4,52 \\
\hline $40^{\circ} \mathrm{C}$ & 0,026 & 26,95 \\
\hline $30^{\circ} \mathrm{C}$ & 0,003 & 205,80 \\
\hline \multicolumn{3}{|c|}{ Músculo blanco de jurel. } \\
\hline Temperatura $\left({ }^{\circ} \mathrm{C}\right)$ & $\mathrm{k}_{\mathrm{i}}\left(\mathrm{h}^{-1}\right)$ & $t_{1 / 2}(h)$ \\
\hline $50^{\circ} \mathrm{C}$ & 0,0467 & 14,83 \\
\hline $40^{\circ} \mathrm{C}$ & 0,0127 & 54,64 \\
\hline $30^{\circ} \mathrm{C}$ & 0,0090 & 81,28 \\
\hline \multicolumn{3}{|c|}{ Surimi de músculo blanco de jurel. } \\
\hline Temperatura $\left({ }^{\circ} \mathrm{C}\right)$ & $k_{i}\left(h^{-1}\right)$ & $t_{1 / 2}(h)$ \\
\hline $50^{\circ} \mathrm{C}$ & 0,0734 & 9,44 \\
\hline $40^{\circ} \mathrm{C}$ & 0,0238 & 29,11 \\
\hline $30^{\circ} \mathrm{C}$ & 0,0226 & 30,72 \\
\hline
\end{tabular}


Tabla 3. Valores de energía de activación de la desnaturalización (Ea) para las distintas transglutaminasas

\begin{tabular}{|l|c|c|}
\hline \multicolumn{1}{|c|}{ Enzima } & $\mathrm{E}_{\mathrm{ia}}(\mathrm{Kcal} / \mathrm{mol})$ & $\mathrm{E}_{\mathrm{ia}}(\mathrm{KJ} / \mathrm{mol})$ \\
\hline \multicolumn{1}{|c|}{ Microbiana } & 5,13 & 21,46 \\
\hline Miofibrillas & 9,41 & 39,37 \\
\hline Músculo de Jurel & 4,16 & 17,41 \\
\hline Surimi & 2,88 & 12,05 \\
\hline
\end{tabular}

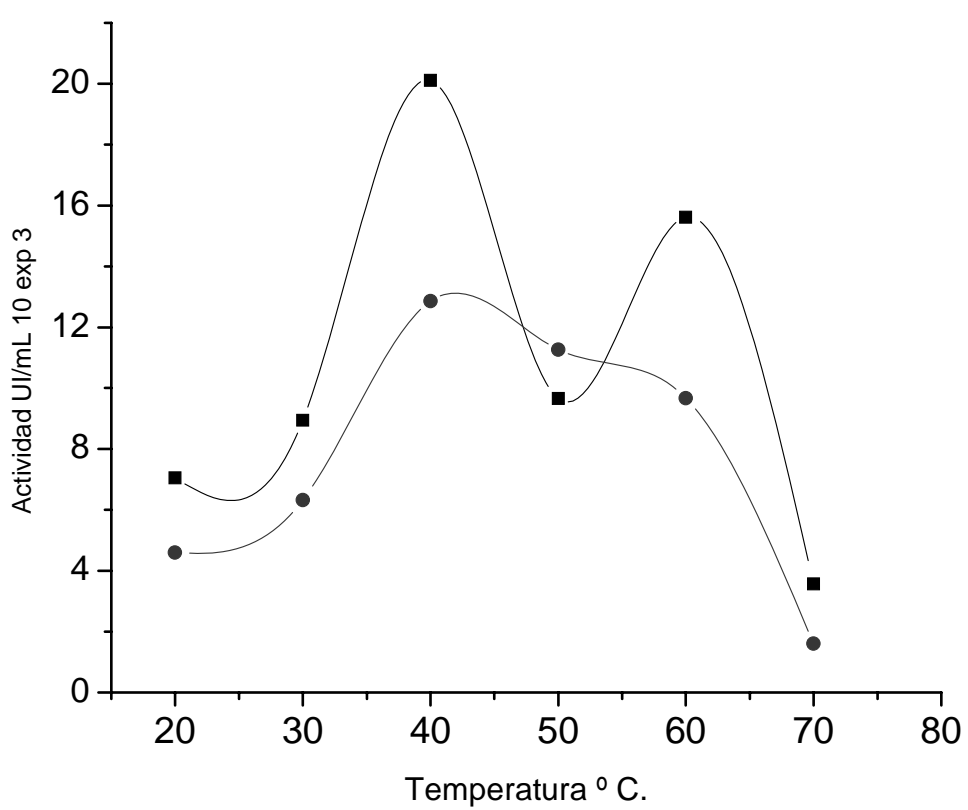

Fig.6 Actividad de transglutaminasa en función de temperatura en extracto crudo purificado de miofibrillas de vacuno analizado con el método de Gauss.

- (extracto crudo), _ • (extracto semipurificado).

\section{CONCLUSIONES}

Se logró caracterizar las transglutaminasas de diferentes orígenes en cuanto temperatura, $\mathrm{pH}$ y efecto del ión $\mathrm{Ca}^{+2}$. Se determinaron las constantes de inactivación para cada uno de las transglutaminasas de diferente origen comparándolas con la transglutaminasa microbiana utilizada industrialmente, encontrándose que la transglutaminasa endógena de miofibrillas de vacuno es la más estable a las temperaturas analizadas. Se determinó la energía de activación de la inactivación para cada una de las transglutaminasas, lo que permite con estos valores obtenidos, estudiar la concentración de transglutaminasa exógena necesaria para la formulación de nuevos productos alimentarios.

\section{AGRADECIMIENTOS.}

Investigación financiada por FONDECYT, Proyecto Nº 1030417

\section{REFERENCIAS}

Ajinomoto Co., Facsimil de Especificaciones de Transglutaminasa Comercial (TG-BH) (1997).

Ando, H., Adachi, M., Umeda, K., Matsuumura, A., Nonaka, M., Uchio, R., Tanaka, H y Motoki, M., Purification and characteristics of a novel transglutaminase derived from microorganism. Agric. Biol. Chem., 53, 2613-2617. (1989).

Asagamil, T., Ogiwara, M., Wakameda, A. y Noguoshil, S. Effect of microbial transglu taminase on quality of frozen surimi made of various kinds of fish species. Fish Sc. 62 (2). 267-272 (1995) 


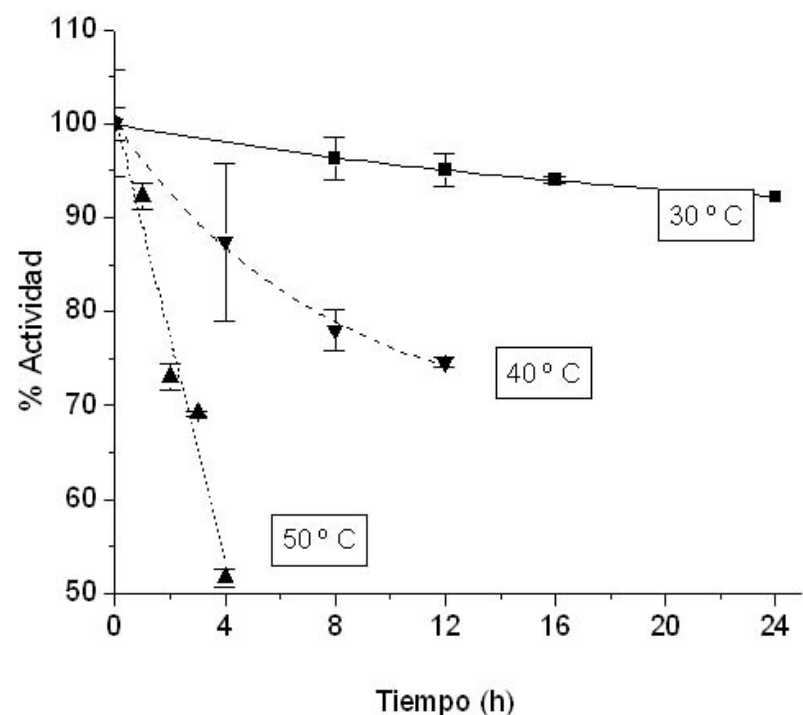

a

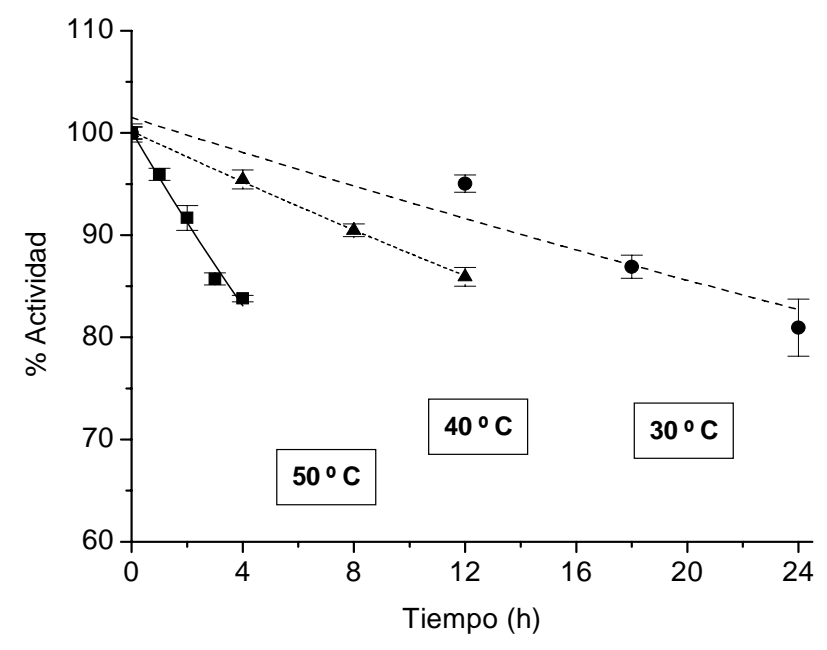

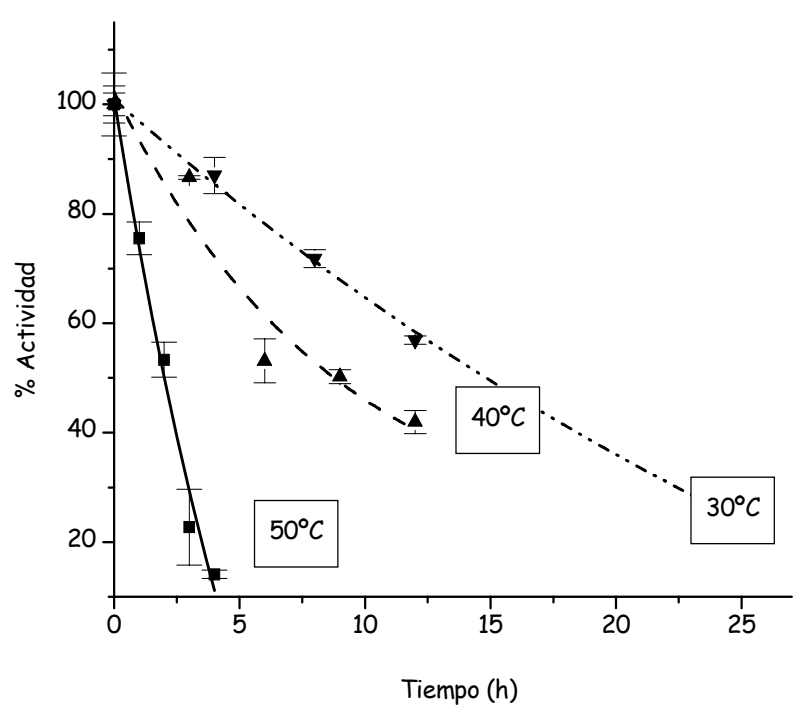

b

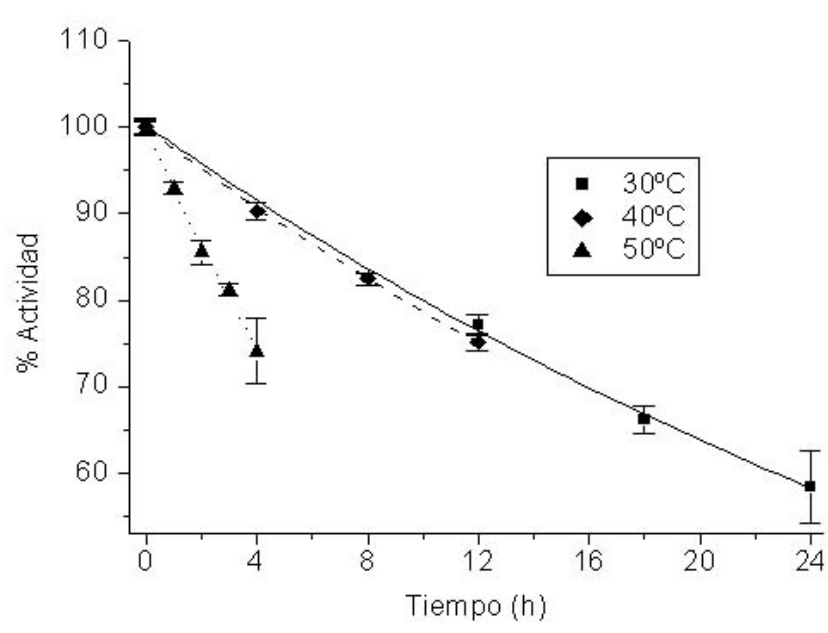

d

Fig. 7: Gráficos de inactivación de transglutaminasa a diferentes tiempos y temperatura. a) Microbiana; b) Miofibrillas de carne de vacuno, c) Músculo blanco de jurel y d) Surimi de músculo blanco de jurel.

Batista, I., Salterio, A-T. y Mateus, M.J., Preliminary Characterization of European Sardine Transglutaminase. Journal of Aquatic Food Product Technology 11,(3/4), 57-64 (2002).

Dondero, M., Curotto, E. y Figueroa, V. Transglutaminase effects on gelation of Jack Mackerel surimi (Trachurus murphyi). Rev. Food Sci. Tech. Int. 8, (1), 49-54 (2002).

Dondero, M., Figueroa, V., Morales, X. y Curotto, E. Transglutaminase effects on gelation capacity of thermally induced beef protein gels. Food Chemistry. 99, (3) 546-554 (2006).

Folk, J.E. y S.I. Chung, Molecular and catalityc properties of transglutaminase. Adv. Enzimolog, 38, 109-191 (1973).

Folk, J.E., Transglutaminases. Ann. Rev. Biochem. 49, 517-531.(1980).

Folk J.E. y J.S. Finlayson, The $\varepsilon$-( $\gamma$-glutamyl)-lysine cross-link and the role of transglutaminase. Advances in protein Chemistry, 31, 1-133. (1997). 
Greco, G., Pirozzi, D., Marenmonti, M., Toscano, G. y Gianfreda, L., The kinetic of enzyme inactivation. 429-435. In: W. Van DenTweel, A., Harder y R Buitelaar (Ed). Stability and Stabilization of enzymes. Elsevier, Amsterdam. (1993).

Icekson, I. y Apelbaum, A., Evidence for transglutaminase activity in plant tissue. Plant Physiol., 84, 972-974. (1987).

Illanes, A., Biotecnología de enzimas. Monografía 35, OEA.,127-128. Ediciones Universitarias de Valparaíso. PUCV. (1994).

Kishi, H., Nozawa, H., y Seki, N., Reactivity of muscle transglutaminase on carp myofibrils and myosin B. Nippon Suisan Gakkaishi. 57, 1203-1210. (1991)

Kumazawa Y., Numazawa, T., Seguro, K. y Motoki., M., Suppression of surimi gel setting by transglutaminase inhibitors. J. Food Sci., 60, 715-717 (1995).

Kumazawa, Y., Nakanishi, K., Yasueda, H., y Motoki, M. Purification and characterization of Transglutaminase from Walleye Pollack Liver. Fisheries Science 62, (6), 959-964. (1996)

Kumazawa, Y. y otros 5 autores, purification and characterization of transglutaminase from Japanese Oyter (Crassostrea gigas). Journal Agric. Food Chem.45, 604-610. (1997).

Laemmli, U., K. y Favre, M., Maturation of head of bacteriophage T4. J. Mol. Biol. 80, 575-599. (1973)

Lee, H., Lanier, T; Hamann, D. y Knopp, J. Transglutaminase effects on low temperature gelation of fish sols. J. Food Sci. 62, (1), 20-24. ( 1997).

Lowry, O., Rosebrough, N., Farr, A. y Randall, R., Protein measurement with the folin Phenol reagment. Biol. Chem. 193; 265-275. (1951).

Nielsen, P., Reaction and potential industrial application transglutaminase: Review of literature and patents. Food Biotech. 9, (3), 119-156 (1995).

Nowsad , A., Katoh, E., Kanoh, S., y Niwa E. Contribution of transglutaminase to setting of fish pastes at various temperatures. Fisk. Sci. 62, (1), 94-97 (1996).

Nury, S., Meunier, J.C. y Mouranche, A., The kinetics of thermal deactivation of transglutaminase from guinea pig liver, Eur. J. Biochem. 180, 161-166 (1989)

Prinal. Transglutaminasa el Ingrediente del futuro. Rev. Técnica, Tecnología y Publicitaria para la Industria Alimentaria. №1. 2000.

Seki, N. y otros 6 autores, Transglutaminase activity in Alaska pollack muscle and surimi, and its reaction with myosin B. Nip. Suisan Gak. 56, (1), 125-132.( 1990).

Tsai, G., Lin, S. y Jiang, S. Transglutaminase from Streptoverticillium ladaka num and application to minced product. J. of Food Sci. 61, (6), 1234-1238. (1996).

WAN, J. y Seki, N. Effects of salts on transglutaminase mediated crosslinking of myosin in suwart gel from Walleye pollock. Nip. Suisan Gak. 58, (11), 2181-2187 (1992).

Wan, J., Kimura, I., Satake, M., y Seki, N. Effect of calcium ion concentration on the gelling properties and transglutaminase activity of Walleye pollack surimi paste. Fisheries Sci., 60, 107-113. (1994).

Yasueda, A. y Kumasawa, Y., Motoki, M. Purification and characterization of a Tissue-type Transglutaminase from Red Sea Bream (Pagrus major). Biotech. Biochem. 58 (11), 2041-2045 (1994).

Zhu, Y., Rinzema, A., Tramper, J., y Bol, J., Microbial transglutaminase. A review of its production and application in food processing. Appl. Microbiol. Biotechnol. 44, 277-282 (1995) 